\title{
Nasal Tip Plasty Using a Batten Graft with Ear Cartilage in East Asians
}

\section{Sang Pil Tae, Jin Kyung Song, Hong Sil Ju, Seong yoon Lim}

Department of Plastic and Reconstructive Surgery, Hanil General Hospital, Seoul, Korea
No potential conflict of interest relevant to this article was reported.
Background Plastic surgeons have constantly investigated methods of nasal tip plasty and nasal lengthening in rhinoplasty. The septal extension graft has been widely applied as a very useful method for East Asians who have different internal nasal structures and nasal skin from those of Caucasians. In this study, we performed nasal tip plasty with a batten-type septal extension graft using ear cartilage.

Methods Nasal tip plasty was performed for 48 patients by batten graft with ear cartilages at the Cocoa Plastic Surgery Clinic and in the plastic surgery unit of Hanil General Hospital from January 2009 to January 2015. A batten-type septal extension graft was constructed with cymba of the concha cartilage. An average area of $2.0 \mathrm{~cm} \times 1.4 \mathrm{~cm}$ of the cymba and $1.0 \mathrm{~cm} \times 1.0 \mathrm{~cm}$ of the cavum from the concha cartilage was harvested. Results Of the 48 patients who underwent batten-type septal extension graft using ear cartilages, nasal tip plasty and columellar lengthening were successfully achieved in almost all of the patients. The nasal tip's height was well-maintained for the follow-up period ranging from 6 months to 5 years after surgery.

Conclusions Nasal tip plasty using a septal extension graft is a very useful method for East Asians with a low nasal tip. The septal extension graft with ear cartilage enables the creation of a desirable nasal shape because there is a sufficient amount of ear cartilage to stably support the nasal tip, thus creating a natural and smooth nose shape.

Keywords Asian group, Ear cartilage, Nasal tip plasty, Rhinoplasty

\section{INTRODUCTION}

In rhinoplasty, methods of nasal tip plasty and nasal lengthening have been consistently studied by plastic surgeons. Many plastic surgeons have used an alloplastic graft with L-shaped silicone for caudal rotation and augmentation of the projection of the nasal dorsum and tip for several decades $[1,2]$. However, nasal tip plasty using an alloplastic graft has a high rate of complications. Therefore, utilizing autologous cartilage is a safe method for performing nasal tip plasty and for derotation of the nasal tip.

Received: May 16, 2016 Revised: Jun 12, 2016 Accepted: Jun 14, 2016 Correspondence: Jin Kyung Song Department of Plastic and Reconstructive Surgery, Hanil General Hospital, 308 Uicheon-ro, Dobong-gu, Seoul 01450, Korea. E-mail: cococoeye@gmail.com

Copyright @ 2016 The Korean Society for Aesthetic Plastic Surgery.

This is an Open Access article distributed under the terms of the Creative Commons Attribution Non-Commercial License (http://creativecommons.org/licenses/by-nc/4.0/) which permits unrestricted non-commercial use, distribution, and reproduction in any medium, provided the original work is properly cited. www.e-aaps.org
Rhinoplasty for East Asians is quite different from that for Caucasians because there are anatomical differences in the internal structure of the nose and nasal skin between East Asians and Caucasians. To be specific, East Asian skin is thicker and less stretchable than Caucasian skin [3]. In addition, East Asian alar cartilage is structurally unstable, so that the nasal tip is supported by ligaments and soft tissues rather than the alar cartilage [4]. Moreover, there is a major difference in the structure of the nasal septum between East Asians and Caucasians. The nasal septum is one of the key structures in the supportive framework of the nasal tip. The nasal septum of East Asians is relatively smaller, thinner, and weaker than that of Caucasians [5]. Therefore, performing soft tissue dissection with sufficient supporting cartilage is required for rhinoplasty of East Asians. The septal extension graft is particularly useful for East Asians because it can function as a support framework for the nose. In addition, it can maintain the nasal septum along the length of the whole nasal structure [6]. However, the septal extension graft in East Asians has the following limitations. It can result in relatively lower height of the nose, unstable alar carti- 
lage, and a small or weak nasal septum. Therefore, the objective of this study was to obtain similar effects and maintain the advantages of the surgical technique by performing the septal extension graft with ear cartilage. Here, we report the positive results of this surgery.

\section{METHODS}

\section{Subjects}

The subjects of this study were 48 patients who underwent nasal tip plasty through septal extension grafts using ear cartilages in Cocoa Plastic Surgery Clinic and the plastic surgery department of Hanil General Hospital from January 2009 to January 2015. All subjects were East Asians with a low nasal tip. We performed the septal extension graft using ear cartilage for nasal tip plasty and nasal dorsal augmentation with silicone implants.

\section{Methods}

\section{Incision and approach}

Open rhinoplasty was performed for all patients. A small V-shaped incision was made across the columella in the thinnest part. With a \#15 knife, a skin incision was made along the direction of the caudal border of the medial crura while taking care not to cause any damage to the medial crura. After that, an internal mucosal incision was made along the caudal border of the lateral cartilage. After performing dissection on the lateral cartilage, the supraperichondrial plane in the middle cartilage was connected in a caudal direction.

\section{Ear cartilage harvesting technique}

In order to obtain ear cartilage, a surgical approach was performed in the anterior ear. The subcutaneous tissue layer was dissected from the supraperichondrial layer while avoiding damage to the perichondrium. With a \#15 knife, a 1.0-mm deep incision was made along the lateral border of the cymba and cavum of the concha car-

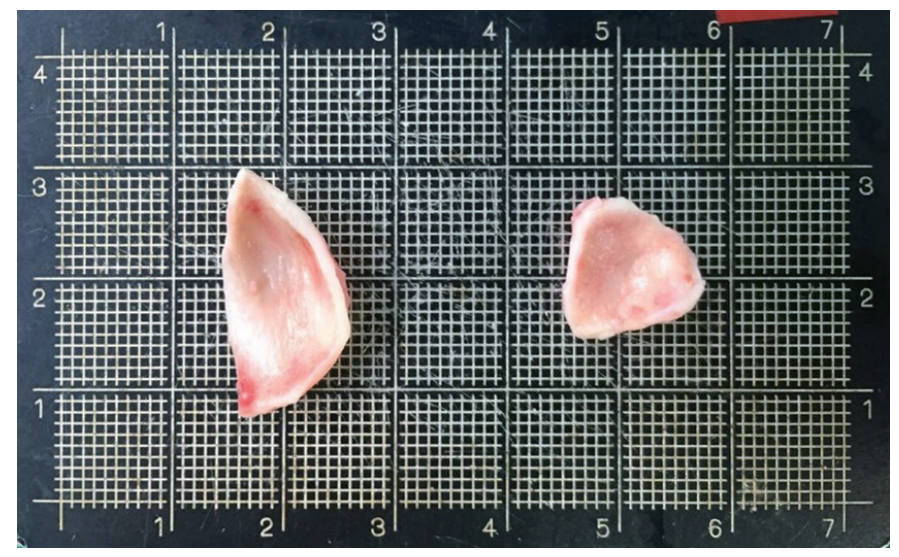

Fig. 1. Harvested ear cartilage from the cymba and cavum concha of the ear cartilage. tilage. The cymba of the concha cartilage was harvested in an oval shape with a mean dimension of $2.0 \mathrm{~cm}(1.8-2.2) \times 1.4 \mathrm{~cm}(1.2-1.6)$ and a mean thickness of $1.2 \mathrm{~mm}$ (1-1.5). After harvesting the cymba of the concha cartilage, a circular-shaped cavum of the concha cartilage $1 \mathrm{~cm}$ in diameter was harvested (Fig. 1). In the process, we set aside at least a 2-mm width of the crus of the helix located between the cymba and cavum of the concha. We then conducted bolster suturing at the harvest site.

\section{Nasal tip grafting technique}

The septal extension graft is located from the nasal tip to the nasal septum of the caudal border. The septal extension graft plays a role in making one's nasal tip rise with the cymba of the concha carti-

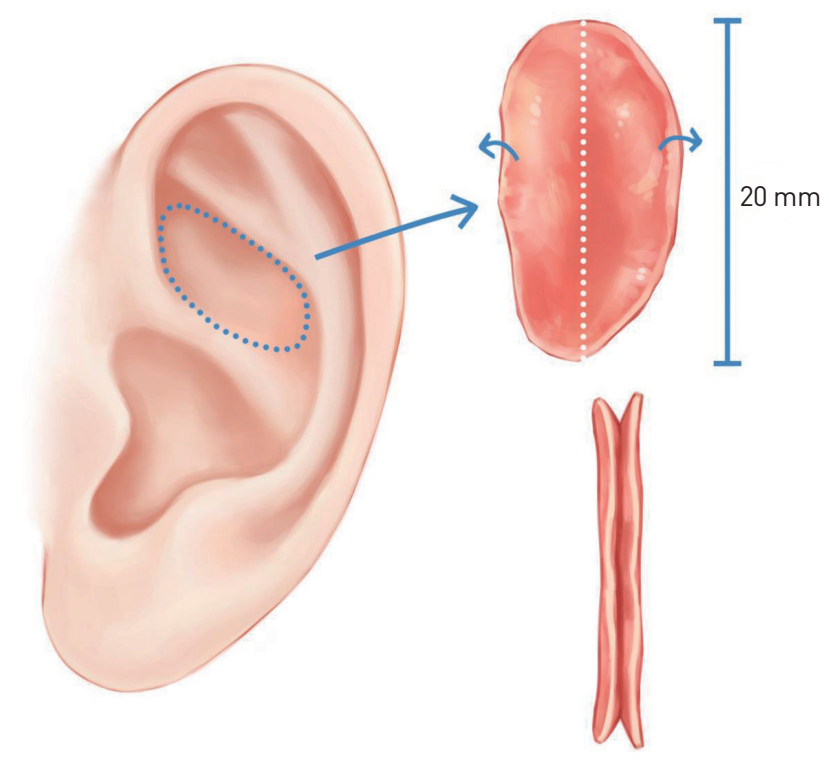

Fig. 2. Cymba of the concha cartilage harvested from the ear.

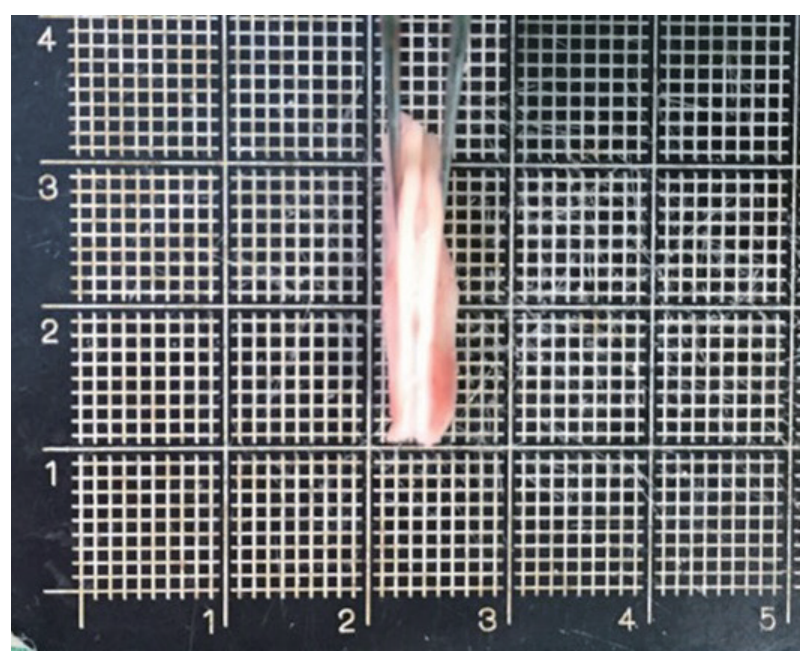

Fig. 3. Cymba of the concha cartilage bent and closed together along its convex side. 
lage. An average dimension of $2.0 \mathrm{~cm} \times 1.4 \mathrm{~cm}$ of the harvested cymba of the concha cartilage is folded on its major axis while closing together its convex sides and avoiding damage to the perichon-

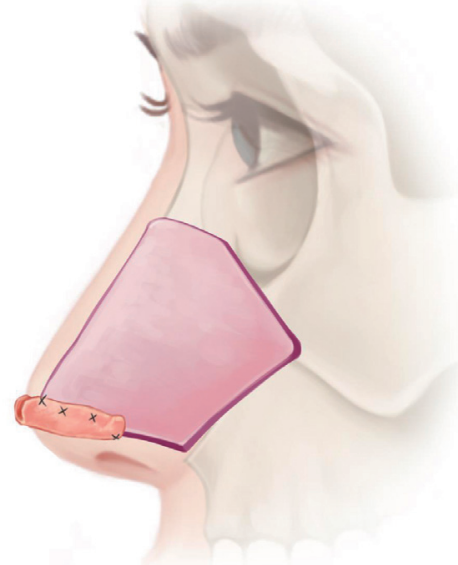

Fig. 4. Septal extension graft of the concha cartilage in batten fashion. Two-point fixation of the septum and additional two-point fixation against the rotation of the graft. drium of the concave sides (Fig. 2 and 3). This graft is placed diagonally across the caudal and dorsal L-strut of the nasal septum. The graft is located beneath the connection between the alar cartilage and the nasal septum. In order to firmly affix the graft to the

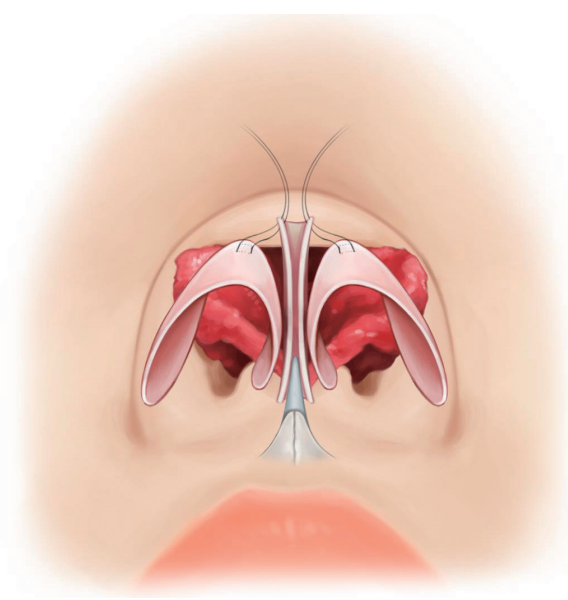

Fig. 5. Interdomal suture to create the nasal tip shape.

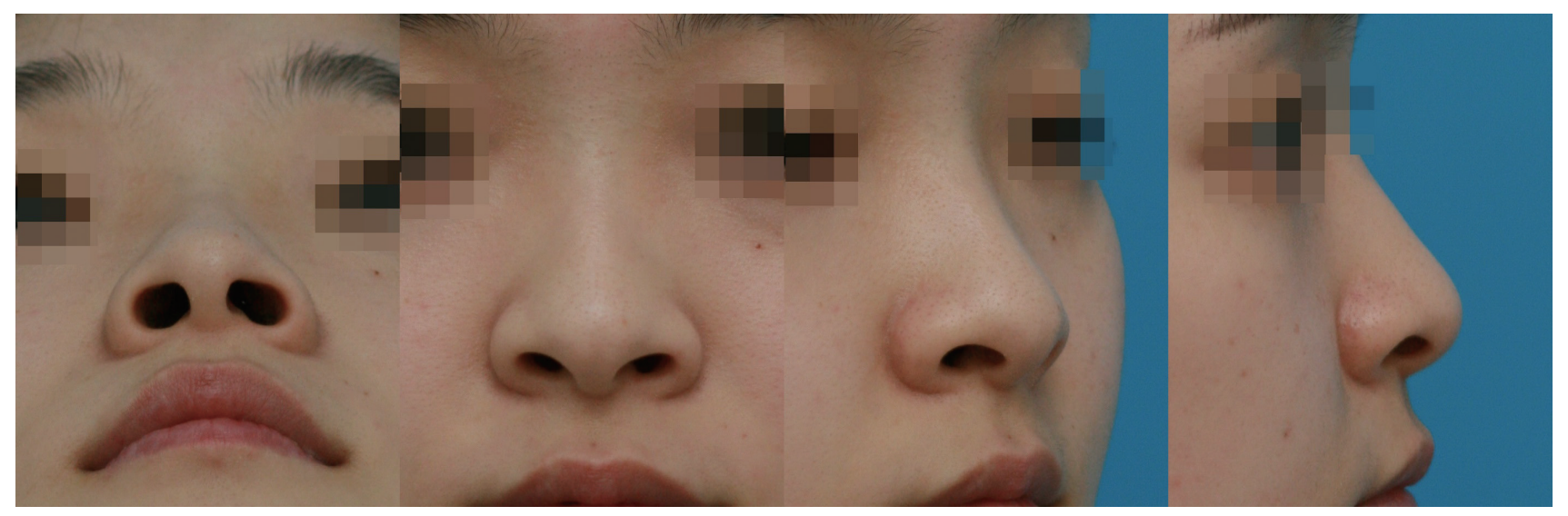

Fig. 6. Preoperative view of 24-year-old woman with a low nasal tip and wide alar base.

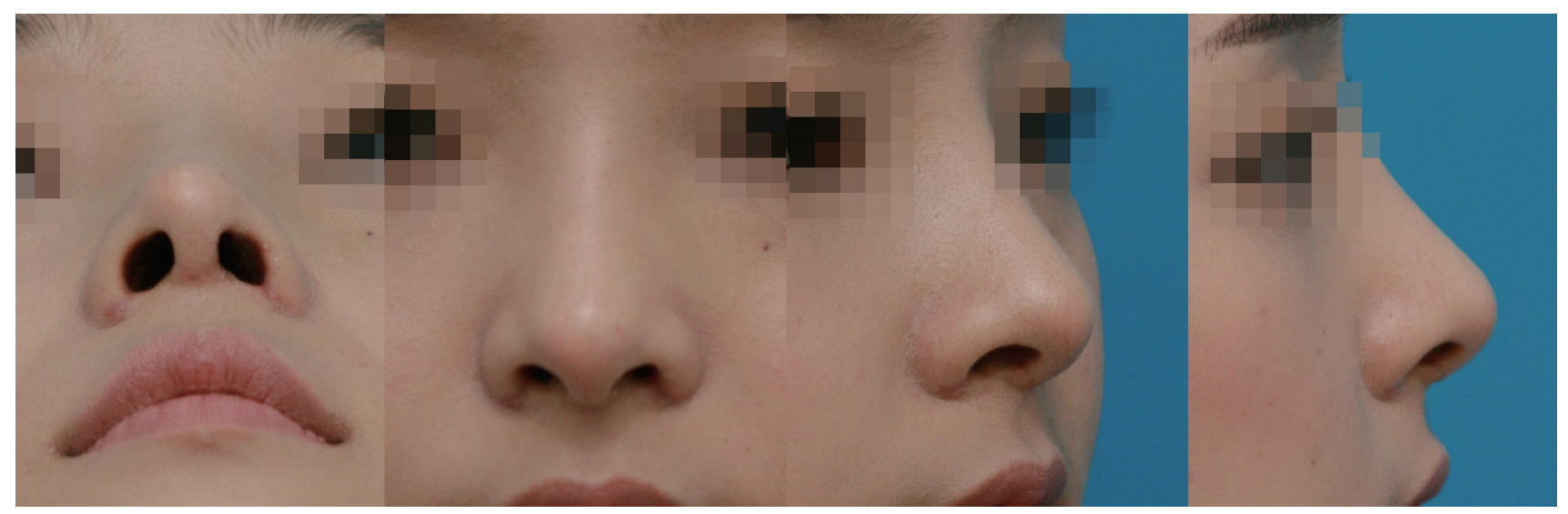

Fig. 7. View six months postoperatively of the patient who underwent a batten-type septal extension graft, onlay graft, and alar base reduction. 
nasal septum cartilage, the overlapping region between the graft and the caudal septum is sutured separately in a figure-eight fashion in two locations (Fig. 4). When we apply the cymba of the concha cartilage, it should project over $3 \mathrm{~mm}$ from the caudal end of the septum. The nasal tip shape is created with interdomal suture techniques after connecting the highest part of the graft to the middle crus of the alar cartilage (Fig. 5).

For the onlay graft, a circular-shaped cavum of the concha cartilage $1 \mathrm{~cm}$ in diameter is used. The harvested cavum of the concha cartilage can be added to enhance the height of the nasal tip.

\section{RESULTS}

All 48 patients underwent septal extension graft using ear cartilages. Nasal tip plasty and columellar lengthening were successfully achieved in almost all of the patients. The height of the nasal tip has been well-maintained in all patients in the follow-up period (from 6 months to 5 years after surgery). Two of the patients complained of slight nasal tip drooping, but revision was not necessary.

\section{Case 1}

The first case was a 24-year-old female patient with the typical nose shape of an East Asian: low and wide ala nasi (Fig. 6). Harvested cymba and cavum of the concha cartilage were used for the septal extension graft and the onlay graft, respectively. Silicone was used for dorsum augmentation. Alar reduction were performed. Tip augmentation and columellar lengthening were well-maintained at 6 months after surgery (Fig. 7).

\section{Case 2}

The second case was a 32-year-old female patient with the typical features of an East Asian nose, including a short nose, short columella, and weak alar cartilage (Fig. 8). Harvested cymba and cavum of the concha cartilages from the ear were used for nasal tip plasty by septal extension graft. The tip augmentation and columellar lengthening were well-maintained at 8 months after the operation (Fig. 9).

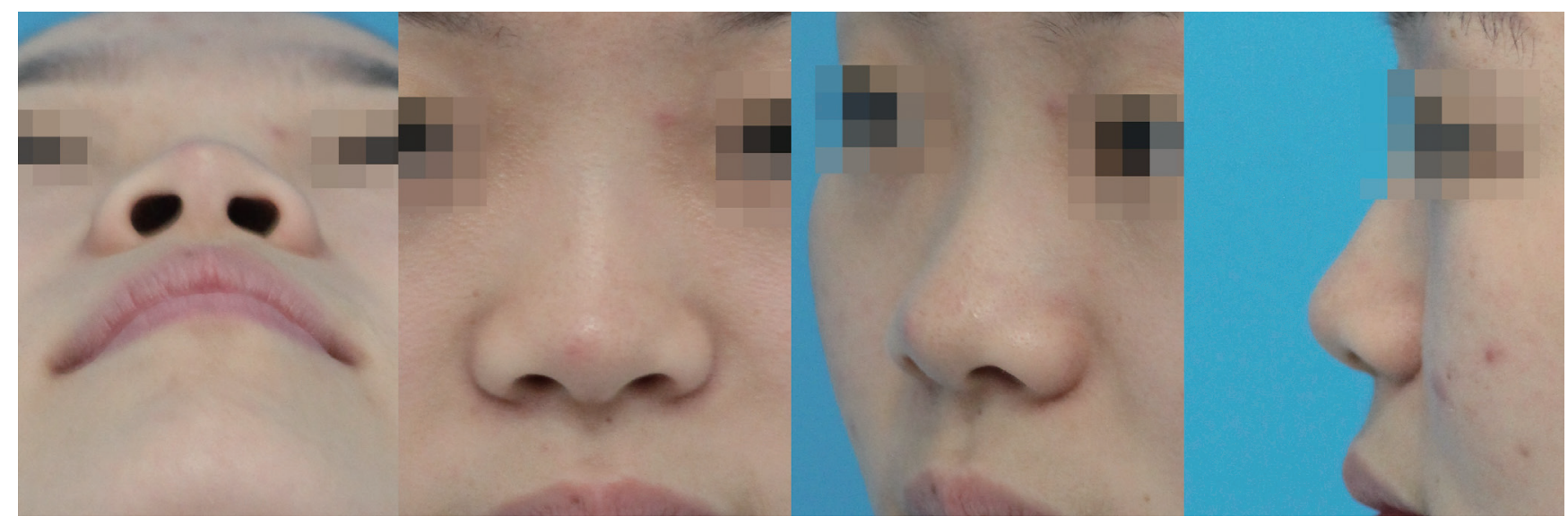

Fig. 8. Preoperative view of a 24-year-old woman who has a short nose, short columella, and weak alar cartilage.

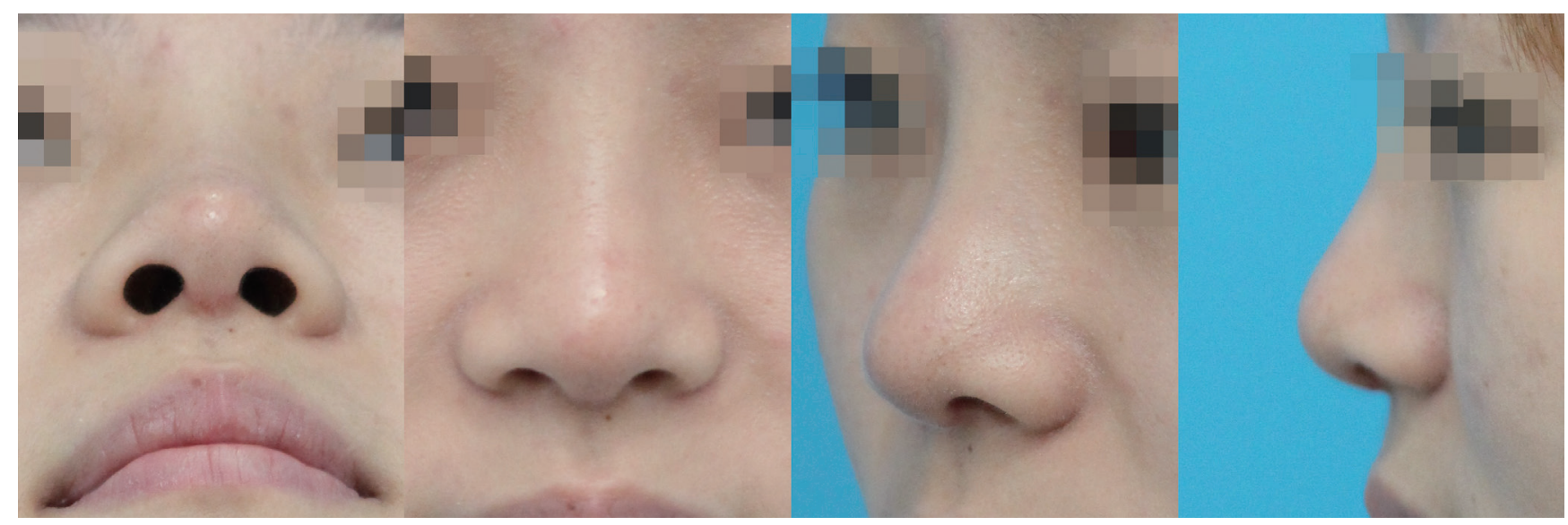

Fig. 9. View 8 months postoperatively of the patient with a well-maintained nasal tip height. 


\section{DISCUSSION}

In rhinoplasty, nasal tip plasty and creating a desirable and attractive nose shape are important factors in determining patient satisfaction with surgery. However, many plastic surgeons have difficulty achieving these goals. This is because the nasal tip is a dynamic structure. It consists of several cartilages, soft tissues, and their connections. In this respect, nasal tip plasty may weaken these supportive structures such that the nose shape after the operation may be deformed or transformed into one outside the expectation of the surgeon. A columellar strut has been commonly performed in the past. However, it is difficult to use a columellar strut to raise the nose to the level that the surgeon or the patient desires. In addition, this technique has a strong possibility of deterioration. The nose can return to the patient's original nose shape, that is, the same as the preoperative state $[7,8]$. Furthermore, it is difficult to use cartilages when the upper lateral cartilage is too short or its width is too narrow or when the mid-vault has already deteriorated, accompanied by the above problems of the upper lateral cartilage [3]. Prospective studies by Rich et al. [9] have noted that most surgical procedures eventually fail to achieve nasal tip augmentation. These studies also concluded that revising the dorsum can function as an illusion, leading the observer to believe that the nasal tip has been elevated [10]. On the other hand, the septal extension graft is outstanding to support and raise the nasal tip without a functional effect on the nasal airway [7].

East Asian noses have different anatomical structures from Caucasian ones. Compared to Caucasian noses, East Asian noses are characterized by a weaker cartilage framework and thicker skin. Because East Asian noses have thicker skin and heavier tension, they have flatter and broader nasal tips compared to Caucasian noses. Moreover, the alar cartilages in East Asian noses are less developed than those of Caucasian ones. Due to the short columella, East Asian noses have depressed flat nasal tips [11]. Byrd et al. [7] have described patients with weak upper lateral cartilage as a highrisk group. This is because rhinoplasty for nasal tip plasty can detach the internal cartilage from the nasal septum and result in weakened nasal tip support. Therefore, Byrd et al. [7] devised the septal extension graft in order to avoid these risks. East Asian noses are more suitable for the septal extension graft than the noses of Caucasians because their nasal cartilage structures are so weak that their nasal tip's supporting structure is unstable.

According to the projection, shape, and rotation of the nasal tip, the methods devised by Byrd et al. [7] are classified into three types of septal extension grafts: the spreader type, batten type, and direct extension type. These three types are classified according to the location where the grafts are placed. In this study, we used the batten type for the septal extension graft. The batten type is located diagonally across the caudal and dorsal L-strut of the nasal septum. Therefore, it has the advantage of not affecting the internal nasal valve and the length of the mid-vault $[7,12]$.

The most common donor site for the septal extension graft is the nasal septum. However, compared to Caucasian noses, the nasal septum in East Asian noses is much thinner, shorter, and weaker. Thus, the septal extension graft for East Asians is more likely to be performed on one side. As a consequence, it may result in the deviation of the nasal tip due to the lack of available cartilages [10]. For these reasons, in this study, we used ear cartilages and harvested them from two locations of the patient's one ear to obtain enough tissue. For the septal extension graft, an average area of $2.0 \mathrm{~cm} \times 1.4$ $\mathrm{cm}$ of the cymba of the concha cartilage was harvested and used after folding it along its major axis while closing together the convex sides. This technique made the curved ear cartilage flat. In addition, the grafts became stronger and harder as the folding sides could support each other. An onlay graft was placed horizontally in the alar cartilage for additional tip augmentation.

When using nasal septum cartilage as a graft, it is difficult to plan ahead for an appropriate size and shape of the cartilage to be used. On the other hand, when ear cartilage is used as a graft, it is possible to predict the size available before surgical procedures and to extract the desired shape of the cartilage. Furthermore, ear cartilages consist of elastic cartilages, whereas nasal septum cartilages are comprised of hyaline cartilages. As a result, ear cartilages are softer and more elastic than nasal septum ones. Therefore, using ear cartilages can prevent a stiff nasal tip. In addition, they can be used to create a natural and attractive nose shape.

However, there are some limitations to using ear cartilage. First, it is difficult to deal with the ear cartilage because of its curved shape. Second, some complications like hematoma or shrinkage of the ear can occur at the donor site. To prevent hematoma, we performed a bolster suture. In one patient, a small amount of hematoma which did not require aspiration occurred when we used this technique. Shrinkage of the ear can occur because of the structural weakness after harvesting the ear cartilages. To prevent this, we preserved at least a 2-mm width of the crus of the helix located between the cymba and cavum of the concha. Finally, if the cymba of the concha is short $(20 \mathrm{~mm})$ or extremely weak, it is not suitable for nasal tip augmentation that exceeds $3.0 \mathrm{~mm}$ from the caudal septal angle. In that case, cartilage from both ears can be harvested to reinforce the nasal cartilage.

\section{CONCLUSIONS}

Nasal tip plasty using a septal extension graft is a common surgical method for East Asians because East Asian noses are lower in height and the size of the nasal septum cartilages commonly used as a donor site are smaller than in Caucasian noses. Using ear cartilages has many advantages. First, the sufficient volume of ear cartilage can allow us to perform septal extension grafts bilaterally. This enables avoiding deviation of the nasal tip while stably supporting the 
nasal tip. It is also possible to identify the amount of cartilage available in advance and plan ahead to harvest the desired shape of the cartilages, thus making it possible to perform a septal extension graft and achieve the desired nasal shape. In addition, since ear cartilages consist of elastic cartilage, the shape of the nasal tip will be more natural and softer than using nasal septum cartilage.

\section{PATIENT CONSENT}

Patients provided written consent for the use of their images.

\section{REFERENCES}

1. Huang J, Liu Y. A modified technique of septal extension using a septal cartilage graft for short-nose rhinoplasty in Asians. Aesthetic Plast Surg 2012;36:1028-38.

2. Kim JS, Han KH, Choi TH, et al. Correction of the nasal tip and columella in Koreans by a complete septal extension graft using an extensive harvesting technique. J Plast Reconstr Aesthet Surg 2007;60:16370.

3. Choi JY, Kang IG, Javidnia H, et al. Complications of septal extension grafts in Asian patients. JAMA Facial Plast Surg 2014;16:169-75.
4. Kim JH, Song JW, Park SW, et al. Effective septal extension graft for Asian rhinoplasty. Arch Plast Surg 2014;41:3-11.

5. Lin J, Chen X, Wang X, et al. A modified septal extension graft for the Asian nasal tip. JAMA Facial Plast Surg 2013;15:362-8.

6. Kim GR, Park K, Kim T. Use of nasal septal bone for septal extension graft after jaw surgery. Plast Reconstr Surg Glob Open 2013;1:e76.

7. Byrd HS, Andochick S, Copit S, et al. Septal extension grafts: a method of controlling tip projection shape. Plast Reconstr Surg 1997;100:9991010.

8. Akkus AM, Eryilmaz E, Guneren E. Comparison of the effects of columellar strut and septal extension grafts for tip support in rhinoplasty. Aesthetic Plast Surg 2013;37:666-73.

9. Rich JS, Friedman WH, Pearlman SJ. The effects of lower lateral cartilage excision on nasal tip projection. Arch Otolaryngol Head Neck Surg 1991;117:56-9.

10. Seyhan A, Ozden S, Ozaslan U, et al. A simplified use of septal extension graft to control nasal tip location. Aesthetic Plast Surg 2007;31: 506-11; discussion 12-3.

11. Park CH, Lee SO, Hong SJ. Use of septal extension grafts in nasal tip plasty. Korean J Otolaryngol-Head Neck Surg 2005;48:1219-23.

12. Kim MH, Choi JH, Kim MS, et al. An introduction to the septal extension graft. Arch Plast Surg 2014;41:29-34. 